\title{
On Invertibility of Functional Operators with Shift in Weighted Hölder Spaces
}

\author{
Anna Tarasenko1, Oleksandr Karelin ${ }^{2}$ \\ ${ }^{1}$ Mathematical Research Center, Hidalgo State University, Pachuca, Mexico \\ ${ }^{2}$ Advanced Research Center on Industrial Engineering, Hidalgo State University, Pachuca, Mexico \\ Email: anataras@uaeh.edu.mx, karelin@uaeh.edu.mx
}

Received 4 October 2014; revised 1 November 2014; accepted 7 November 2014

Copyright (C) 2014 by authors and Scientific Research Publishing Inc.

This work is licensed under the Creative Commons Attribution International License (CC BY).

http://creativecommons.org/licenses/by/4.0/

(c) (i) Open Access

\begin{abstract}
In this paper, we consider functional operators with shift in weighted Hölder spaces. We present the main idea and the scheme of proof of the conditions of invertibility for these operators. As an application, we propose to use these results for solution of equations with shift which arise in the study of cyclic models for natural systems with renewable resources.
\end{abstract}

\section{Keywords}

Functional Operator with Shift, Hölder Space, Conditions of Invertibility, Renewable Resources

\section{Introduction}

The interest towards the study of functional operators with shift was stipulated by the development of solvability theory and Fredholm theory for some classes of linear operators, in particular, singular integral operators with Carleman and non-Carleman shift [1]-[3]. Conditions of invertibility for functional operators with shift in weighted Lebesgue spaces were obtained [1].

Our study of functional operators with shift in Hölder spaces with weight has an additional motivation: on modeling systems with renewable resources, equations with shift arise [4] [5], and the theory of linear functional operators with shift is the adequate mathematical instrument for the investigation of such systems.

In Section 2, some auxiliary lemmas are proposed. These are to be used in the proof of invertibility conditions. In Section 3, conditions of invertibility for functional operators with shift in Hölder spaces with power wight are obtained. We provide the main idea and the scheme of proof of the conditions of invertibility. At the end of the article, an application to modeling systems with renewable resources is specified.

\section{Auxiliary Lemmas}

We introduce [6] weighted Hölder spaces $H_{\mu}^{0}(J, \rho)$, in which we consider functional operators with shift. 
A function $\varphi(x)$ that satisfies the following condition on $J=[0,1]$,

$$
\left|\varphi\left(x_{1}\right)-\varphi\left(x_{2}\right)\right| \leq C\left|x_{1}-x_{2}\right|^{\mu}, \quad x_{1} \in J, x_{2} \in J, \mu \in(0,1),
$$

is called Hölder's function with exponent $\mu$ and constant $C$ on $J$.

Let $\rho$ be a power function which has zeros at the endpoints $x=0, \quad x=1$ :

$$
\rho(x)=(x-0)^{\mu_{0}}(1-x)^{\mu_{1}}, \quad \mu<\mu_{0}<1+\mu, \mu<\mu_{1}<1+\mu .
$$

The functions that become Hölder functions and turn into zero at the points $x=0, x=1$, after being multiplied by $\rho(x)$, form a Banach space: $H_{\mu}^{0}(J, \rho), J=[0,1]$. The norm in the space $H_{\mu}^{0}(J, \rho)$ is defined by

$$
\|f(x)\|_{H_{\mu}^{0}(J, \rho)}=\|\rho(x) f(x)\|_{H_{\mu}(J)},
$$

where

$$
\|\rho(x) f(x)\|_{H_{\mu}(J)}=\|\rho(x) f(x)\|_{C}+\|\rho(x) f(x)\|_{\mu},
$$

and

$$
\|\rho(x) f(x)\|_{C}=\max |\rho(x) f(x)|,\|\rho(x) f(x)\|_{\mu}=\sup _{x_{1}, x_{2} \in J, x_{1} \neq x_{2}} \frac{\left|\rho\left(x_{1}\right) f\left(x_{1}\right)-\rho\left(x_{2}\right) f\left(x_{2}\right)\right|}{\left|x_{1}-x_{2}\right|^{\mu}} .
$$

We denote by $\mathcal{B}(H)$ the set of all bounded linear operators mapping the Banach space $H$ into the Banach space $H$. The norm of an operator $\mathcal{A} \in \mathcal{B}(H)$ we will denote by $\|\mathcal{A}\|_{\mathcal{B}(H)}$.

Let $\beta(x)$ be a bijective orientation-preserving displacement on $J$ : if $x_{1}<x_{2}$, then $\beta\left(x_{1}\right)<\beta\left(x_{2}\right)$ for any $x_{1} \in J, x_{2} \in J$; and let $\beta(x)$ have only two fixed points: $\beta(0)=0, \beta(1)=1, \beta(x) \neq x$, when $x \neq 0, x \neq 1$. In addition, let $\beta(x)$ be a differentiable function, let $\frac{\mathrm{d}}{\mathrm{d} x} \beta(x) \neq 0$, and let $\frac{\mathrm{d}}{\mathrm{d} x} \beta(x)$ belong to $H_{\mu}(J)$.

Without loss of generality, we assume that for any fixed $x \in(0,1), \lim _{m \rightarrow+\infty} \beta_{m}(x)=0, \lim _{m \rightarrow+\infty} \beta_{-m}(x)=1$ is fulfilled; we have $\beta^{\prime}(0) \leq 1$ and $\beta^{\prime}(1) \geq 1$.

We will use the following notation,

$$
\begin{gathered}
r=\mu_{0}-\mu, s=\mu_{1}-\mu, \rho_{r, s}(x)=x^{r}(1-x)^{s}, \quad \rho_{\mu, \mu}(x)=\rho_{\mu}(x)=x^{\mu}(1-x)^{\mu}, \\
\rho_{r, s ; j}(x)=\rho_{r, s}\left[\beta_{j}(x)\right], \rho_{\mu ; j}(x)=\rho_{\mu, \mu ; j}(x), \beta\left(x_{1}, x_{2}\right)=\frac{\beta\left(x_{1}\right)-\beta\left(x_{2}\right)}{x_{1}-x_{2}} .
\end{gathered}
$$

Lemma 1. $(\forall \beta(x), x \in J)(\forall \varepsilon>0)\left(\exists n_{0} \in \mathbf{N}\right)(\forall x \in J)\left(\exists n_{1}, n_{2} \in \mathbf{N}, n_{1}<n_{2}, n_{0}=n_{2}-n_{1}\right)$

$$
\left[\beta_{n}(x) \in[0, \varepsilon] \cup[1-\varepsilon, 1], n \in \mathbf{N} \backslash\left[n_{1}, n_{2}\right]\right] .
$$

An essential point is that $n_{0}=n_{2}-n_{1}$ is independent from $x$.

Proof. This lemma follows from the properties of shift $\beta(x)$.

Lemma 2. If the following condition is fulfilled,

$$
a(x) \neq 0 ; \quad\left|\beta^{\prime}(0)\right|^{-\mu_{0}+\mu}\left|\frac{b(0)}{a(0)}\right|<1,\left|\beta^{\prime}(1)\right|^{-\mu_{1}+\mu}\left|\frac{b(1)}{a(1)}\right|<1,
$$

then the following inequalities are correct in some $\varepsilon$ half-neighborhoods of the endpoints $x=0, x=1$ :

$$
\left|u(x) \frac{\rho_{r, s}(x)}{\rho_{r, s ; 1}(x)}\right| \leq q<1, \quad x \in[0, \varepsilon] \cup[1-\varepsilon, 1] .
$$

Proof. This lemma follows from (1) and from the properties of $\beta(x), a(x), b(x)$.

From Lemma 1 and Lemma 2 it follows that for $\varepsilon>0$ a positive integer $n_{0}$ exists such that for any 
$x \in[0,1]$ at most $n_{0}$ values of $\beta_{n}(x)$ will be outside of $[0, \varepsilon] \cup[1-\varepsilon, 1]$.

The following lemmas hold.

Lemma 3. Operator $B_{\beta}$ is bounded in space $H_{\mu}(J),\left\|B_{\beta}\right\|_{\mathcal{B}\left(H_{\mu}(J)\right)} \leq\left\|\beta^{\prime}\right\|_{C}^{\mu}$.

Operator $B_{\beta}$ is bounded in space $H_{\mu}^{0}(J, \rho),\left\|B_{\beta}\right\|_{\mathcal{B}\left(H_{\mu}^{0}(J, \rho)\right)} \leq\left\|\frac{\rho}{\rho[\beta]}\right\|_{H_{\mu}(J)}\left\|B_{\beta}\right\|_{\mathcal{B}\left(H_{\mu}(J)\right)}$.

Lemma 4. For $\varphi \in H_{\mu}^{0}(J, \rho)$, the inequality $\left\|\frac{\rho \varphi}{\rho_{\mu, \mu}}\right\|_{C} \leq 2^{\mu}\|\varphi\|_{H_{\mu}^{0}(J, \rho)}$ is correct.

We shall take advantage of these lemmas in the proof of invertibility conditions in Section 3.

\section{Conditions of Invertibility for Operator $A$ in Weighted Hölder Spaces}

In weighted Hölder space $H_{\mu}^{0}(J, \rho)$, the operators: $A=a I-b B_{\beta}$, where $a \in H_{\mu}, b \in H_{\mu}$, and $U=I-u B_{\beta}$, where $u=b / a$, are invertible simultaneously when $a \neq 0$. It is obvious that $A=a U$ and $A^{-1}=U^{-1} \frac{1}{a} I$.

If a certain natural number $n$ exists such that $\left\|\left(\prod_{j=0}^{n-1} u_{j}(x)\right) B_{\beta}^{n}\right\|_{\mathcal{B}\left(H_{\mu}^{0}(J, \rho)\right)}<1$, where $u_{j}(x)=u\left[\beta_{j}(x)\right]$, then operator $U$ is invertible in space $H_{\mu}^{0}(J, \rho)$ and

$$
U^{-1}=\left(I+u B_{\beta}+\cdots+\left(\prod_{j=0}^{n-2} u_{j}(x)\right) B_{\beta}^{n-1}\right)\left(I-\left(\prod_{j=0}^{n-1} u_{j}(x)\right) B_{\beta}^{n}\right)^{-1} .
$$

This statement in weighted Lebesgue spaces was proved in [1]. The proof is completely transferred without change to the weighted Hölder space as the applied algebraic operations do not depend on the specific properties of the spaces.

Analogously, if $b \neq 0$ and a certain natural number $n$ exists such that $\left\|\left(\prod_{j=0}^{n-1} v_{j}(x)\right) B_{\beta}^{-n}\right\|_{\mathcal{B}\left(H_{\mu}^{0}(J, \rho)\right)}<1$, where $v(x)=\frac{a\left[\beta^{-1}(x)\right]}{b\left[\beta^{-1}(x)\right]}, v_{j}(x)=v\left[\beta_{j}^{-1}(x)\right]$, then the operator $V=I-v B_{\beta}^{-1}$ is invertible in space $\mathcal{B}\left(H_{\mu}^{0}(J, \rho)\right)$ and its inverse operator is $V^{-1}=\left(I+v B_{\beta}^{-1}+\cdots+\left(\prod_{j=0}^{n-2} v_{j}(x)\right) B_{\beta}^{-n+1}\right)\left(I-\left(\prod_{j=0}^{n-1} v_{j}(x)\right) B_{\beta}^{-n}\right)^{-1}$.

It is obvious that $A=-b B_{\beta}\left[I-\left(B_{\beta}^{-1} \frac{a}{b}\right) B_{\beta}^{-1}\right], A^{-1}=-V^{-1} B_{\beta}^{-1}\left(\frac{1}{b}\right) I$.

We will use the following notation

$$
f_{n}(x)=\left(B_{\beta}^{n} f\right)(x), \beta_{n}^{\mu}\left(x_{1}, x_{2}\right)=\left|\frac{\beta_{n+1}\left(x_{1}\right)-\beta_{n+1}\left(x_{2}\right)}{\beta_{n}\left(x_{1}\right)-\beta_{n}\left(x_{2}\right)}\right|, \quad \tilde{u}_{j}(x)=\frac{\rho_{j}(x)}{\rho_{j+1}(x)} u_{j}(x),
$$

Theorem 1. From conditions (1) it follows that such $n$ exists for which $\left\|\left(\prod_{j=0}^{n-1} u_{j}\right) B_{\beta}^{n}\right\|_{H_{\mu}^{0}(J, \rho)}<1$.

Proof. In order to prove

$$
\left\|\rho\left(\prod_{j=0}^{n-1} u_{j}\right) B_{\beta}^{n} \varphi\right\|_{C}+\left\|\rho\left(\prod_{j=0}^{n-1} u_{j}\right) B_{\beta}^{n} \varphi\right\|_{\mu}<\|\varphi\|_{H_{\mu}^{0}(J, \rho)},
$$


we estimate every summand separately, starting with the first

$$
\left\|\rho\left(\prod_{j=0}^{n-1} u_{j}\right) B_{\beta}^{n} \varphi\right\|_{C} \leq\left\|\rho_{\mu, \mu} \prod_{j=0}^{n-1} u_{j} \frac{\rho_{r, s ; j}}{\rho_{r, s ; j+1}}\right\|_{C}\left\|\rho_{r, s} \varphi\right\|_{C} \leq\left\|\rho_{\mu, \mu} \prod_{j=0}^{n-1} u_{j} \frac{\rho_{r, s ; j}}{\rho_{r, s ; j+1}}\right\|_{C} 2^{\mu}\|\varphi\|_{H_{\mu}^{0}(J, \rho)} .
$$

We took into account $\frac{\rho_{r, s}}{\rho_{r, s ; n}}=\prod_{j=0}^{n-1} \frac{\rho_{r, s ; j}}{\rho_{r, s ; j+1}},\left\|B_{\beta}^{n}\left(\rho_{r, s} \varphi\right)\right\|_{C}=\left\|\left(\rho_{r, s} \varphi\right)\right\|_{C}$, Lemma 3 and Lemma 4.

From (2) of Lemma 2, it follows that the first factor on the right side of inequality (4): $\left\|\rho_{\mu, \mu} \prod_{j=0}^{n-1} u_{j} \frac{\rho_{r, s ; j}}{\rho_{r, s ; j+1}}\right\|_{C}$ tends to zero when $n \rightarrow \infty$.

Now, we estimate the second summand of (3). The following estimate holds

$$
\begin{aligned}
& \left\|\rho\left(\prod_{j=0}^{n-1} u_{j}\right) B_{\beta}^{n} \varphi\right\|_{\mu} \\
& \leq\|\tilde{u}\|_{\mu}\left\|\frac{\rho_{n} \varphi_{n}}{\rho_{\mu ; n}}\left|\sup _{C}\right| \rho_{\mu ; 1}\left(x_{1}\right) \prod_{j=0}^{n-2} \tilde{u}_{j+1}\left(x_{1}\right) \frac{\rho_{\mu ; j+2}\left(x_{1}\right)}{\rho_{\mu ; j+1}\left(x_{1}\right)}\left|+\|\tilde{u}\|_{\mu}\left\|\rho_{n} \varphi_{n}\right\| \sup _{x_{1}<x_{2}}\right| \prod_{j=0}^{n-2} \tilde{u}_{j}\left(x_{2}\right) \beta_{j}^{\mu}\left(x_{1}, x_{2}\right) \mid\right. \\
& \quad+\|\tilde{u}\|_{\mu}\left\|\frac{\rho_{n} \varphi_{n}}{\rho_{\mu ; n}}\right\| \sup _{x_{1}<x_{2}}\left|\sum_{j=0}^{n-3} \rho_{\mu ; j+2}\left(\prod_{i=j}^{n-3} \tilde{u}_{i+2}\left(x_{1}\right) \frac{\rho_{\mu ; i+3}\left(x_{1}\right)}{\rho_{\mu ; i+2}\left(x_{1}\right)} \prod_{k=0}^{j} \tilde{u}_{k}\left(x_{2}\right) \beta_{k}^{\mu}\left(x_{1}, x_{2}\right)\right)\right| \\
& +\|\rho \varphi\|_{\mu} \sup _{x_{1}<x_{2}} \prod_{j=0}^{n-1} \tilde{u}_{j}\left(x_{2}\right) \beta_{j}^{\mu}\left(x_{1}, x_{2}\right) \mid .
\end{aligned}
$$

From Lemma 1 and (2) of Lemma 2, it follows that only $n_{0}$ values of $\beta_{l}\left(x_{1}\right)$ may be outside of the set $[0, \varepsilon] \cup[1-\varepsilon, 1]$, where inequality $\left|\tilde{u}_{l+1}\left(x_{1}\right) \frac{\rho_{\mu ; j}\left(x_{1}\right)}{\rho_{\mu ; l+1}\left(x_{1}\right)}\right| \leq q<1$ holds. Here the number $n_{0}$ is from Lemma 1 .

From (2) of Lemma 2 and the identity

$$
\left|\tilde{u}_{l}\left(x_{2}\right) \beta_{l}^{\mu}\left(x_{1}, x_{2}\right)\right|=\left|\frac{\beta\left(x_{1}\right)-\beta\left(x_{2}\right)}{x_{1}-x_{2}}\right|^{\mu}\left|\frac{x_{2}}{\beta\left(x_{2}\right)}\right|^{\mu}\left|\frac{1-x_{2}}{1-\beta\left(x_{2}\right)}\right|^{\mu}\left|u\left(x_{2}\right) \frac{\rho_{r, s}\left(x_{2}\right)}{\rho_{r, s ; 1}\left(x_{2}\right)}\right|
$$

it follows that some number $\varepsilon_{1}>0$ exists such that $\left|\tilde{u}_{l}\left(x_{2}\right) \beta_{l}^{\mu}\left(x_{1}, x_{2}\right)\right| \leq q_{1}<1$ is fulfilled for all fixed $x_{1}, x_{2}$, $x_{1}<x_{2}$ with the possible exception of $n_{1}$ values of $l$.

All expressions with sup from (5) tend to zero when $n \rightarrow \infty$.

Thus, such $n$ exists that $\left\|\left(\prod_{j=0}^{n-1} u_{j}\right) B_{\beta}^{n} \varphi\right\|_{H_{\mu}^{0}(J, \rho)}<\|\varphi\|_{H_{\mu}^{0}(J, \rho)}$, which means that operator $U=I-u B_{\beta}$ is invertible in space $H_{\mu}^{0}(J, \rho)$.

We will now formulate and prove conditions of invertibility for operator $A$ in the space of Hölder class functions with weight. In [4] these conditions were only formulated but not proved.

Theorem 2. Operator A acting in Banach space $H_{\mu}^{0}(J, \rho)$, is invertible if the following condition is fulfilled:

$$
\sigma_{\beta}[a(x), b(x)] \neq 0, \quad x \in J,
$$

where function $\sigma_{\beta}$ is defined by:

$$
\sigma_{\beta}[a(x), b(x)]=\left\{\begin{array}{l}
a(x), \text { when }|a(i)|>\left[\beta^{\prime}(i)\right]^{-\mu_{i}+\mu}|b(i)|, i=0,1 \\
b(x), \text { when }|a(i)|<\left[\beta^{\prime}(i)\right]^{-\mu_{i}+\mu}|b(i)|, i=0,1 \\
0 \quad \text { in other cases. }
\end{array}\right.
$$


Proof. We consider the following case: $\left\{\begin{array}{l}a(x) \neq 0, x \in J, \\ |a(i)|>\left|\beta^{\prime}(i)\right|^{-\mu_{i}+\mu} \cdot|b(i)|, \quad i=0,1 .\end{array}\right.$

In space $H_{\mu}^{0}(J, \rho)$, operators $a I-b B_{\beta}$ and $U=I-u B_{\beta}$, where $u=b / a$, are invertible simultaneously.

Thus, such $n$ exists that

$$
\left\|\left(\prod_{j=0}^{n-1} u_{j}\right) B_{\beta}^{n} \varphi\right\|_{H_{\mu}^{0}(J, \rho)}<\|\varphi\|_{H_{\mu}^{0}(J, \rho)},
$$

which means that operator $U=I-u B_{\beta}$ is invertible in space $H_{\mu}^{0}(J, \rho)$.

$$
\text { The case }\left\{\begin{array}{l}
b(x) \neq 0, \quad x \in J, \\
|a(i)|<\left|\beta^{\prime}(i)\right|^{-\mu_{i}+\mu} \cdot|b(i)|, \quad i=0,1,
\end{array}\right. \text { can be considered analogously. }
$$

Now, we will focus on the application of these results to the modeling of systems with renewable resources. For the study of such systems, cyclic models were elaborated based on functional operators with shift [4]. The balance relation describing the state of cyclic equilibrium is the equation $a I v-b B_{\beta} v=g$ for the unknown distribution function $v$ which is sought in space $H_{\mu}^{0}(J, \rho)$. In [5], a reproductive summand has been added for a more accurate description of the process of reproduction; this term has been expressed by integrals with degenerate kernels.

If we model the behavior of a system with two resources, taking into account the interaction between them, by integrals with degenerate kernels and following the principles of modeling from [4], we shall obtain two equations with two unknowns, $v_{1}$ and $v_{2}$ :

$$
\begin{aligned}
& a_{1}(x) v_{1}(x)-b_{1}(x) v_{1}\left[\beta_{1}(x)\right]+\Sigma_{1}(x)+\Gamma_{1}(x)=g_{1}(x), \\
& a_{2}(x) v_{2}(x)-b_{2}(x) v_{2}\left[\beta_{2}(x)\right]+\Sigma_{2}(x)+\Gamma_{2}(x)=g_{2}(x),
\end{aligned}
$$

where $v_{1}$ and $v_{2}$ are the densities of the distributions of the first and second resources by their respective individual parameters such as weight or length and

$$
\begin{aligned}
& \Sigma_{1}(x)=\iint_{J} \sum_{i=1}^{m_{1}} \zeta_{1, i}(x) \xi_{1, i}(t) v_{1}(t) \mathrm{d} t, \\
& \Gamma_{1}(x)=\iint_{J}^{n_{1}} \rho_{1, i}(x) \delta_{1, i}(t) v_{2}(t) \mathrm{d} t, \\
& \Sigma_{2}(x)=\int_{J}^{m_{2}} \zeta_{i=1}(x) \xi_{2, i}(t) v_{2}(t) \mathrm{d} t, \\
& \Gamma_{2}(x)=\int_{J}^{n_{2}} \rho_{2, i}(x) \delta_{2, i}(t) v_{1}(t) \mathrm{d} t,
\end{aligned}
$$

are the terms of reproduction and interaction process respectively.

Let $H_{\mu}^{0}(J, \rho)$ be the space in which our model is considered. Suppose that for

$$
A_{1}=a_{1}(x) v_{1}(x)-b_{1}(x) v_{1}\left[\beta_{1}(x)\right], \quad A_{2}=a_{2}(x) v_{2}(x)-b_{2}(x) v_{2}\left[\beta_{2}(x)\right]
$$

in $H_{\mu}^{0}(J, \rho)$ the conditions of invertibility of Theorem 2 are fulfilled. Thus, the inverse operators of $A_{1}$ and $A_{2}$ exist, $A_{1}^{-1}$ and $A_{2}^{-1}$. We apply these inverse operators to the left side of Equation (6) and obtain Fredholm equations of the second type with degenerate kernels. Using a known method of solving such equations, we can find densities $v_{1}$ and $v_{2}$ of the cyclic equilibrium of the system.

\section{References}

[1] Karlovich, Y.I. and Kravchenko, V.G. (1981) Singular Integral Equations with Non-Carleman Shift on an Open Contour. Differential Equations, 17, 2212-2223.

[2] Litvinchuk, G.S. (2000) Solvability Theory of Boundary Value Problems and Singular Integral Equations with Shift. 
Kluwer Academic Publishers, Dordrecht, Boston, London. http://dx.doi.org/10.1007/978-94-011-4363-9

[3] Kravchenko, V.G. and Litvinchuk, G.S. (1994) Introduction to the Theory of Singular Integral Operators with Shift. Kluwer Academic Publishers, Dordrecht, Boston, London. http://dx.doi.org/10.1007/978-94-011-1180-5

[4] Tarasenko, A., Karelin, A., Lechuga, G.P. and Hernández, M.G. (2010) Modelling Systems with Renewable Resources Based on Functional Operators with Shift. Applied Mathematics and Computation, 216, 1938-1944. http://dx.doi.org/10.1016/j.amc.2010.03.023

[5] Karelin, O., Tarasenko, A. and Hernández, M.G. (2013) Application of Functional Operators with Shift to the Study of Renewable Systems When the Reproductive Processed Is Described by Integrals with Degenerate Kernels. Applied Mathematics (AM), 4, 1376-1380. http://dx.doi.org/10.4236/am.2013.410186

[6] Duduchava, R.V. (1973) Unidimensional Singular Integral Operator Algebras in Spaces of Holder Functions with Weight. Proceedings of A. Razmadze Mathematical Institute, 43, 19-52. (In Russian) 
Scientific Research Publishing (SCIRP) is one of the largest Open Access journal publishers. It is currently publishing more than 200 open access, online, peer-reviewed journals covering a wide range of academic disciplines. SCIRP serves the worldwide academic communities and contributes to the progress and application of science with its publication.

Other selected journals from SCIRP are listed as below. Submit your manuscript to us via either submit@scirp.org or Online Submission Portal.
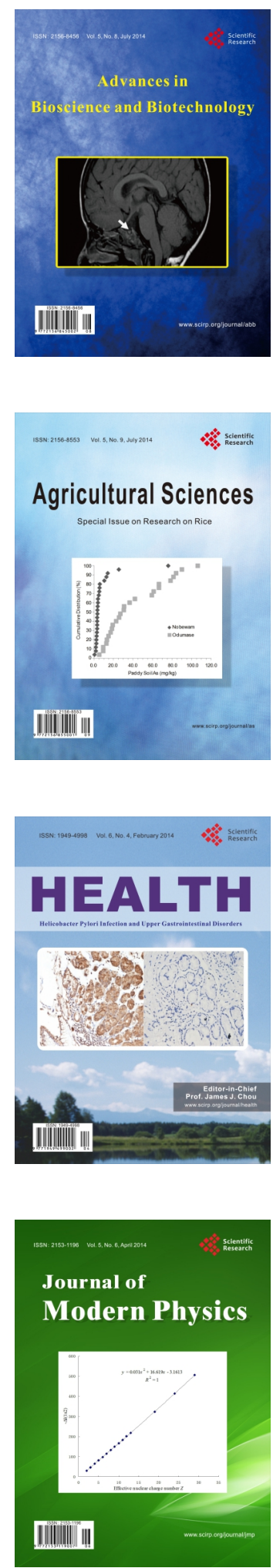
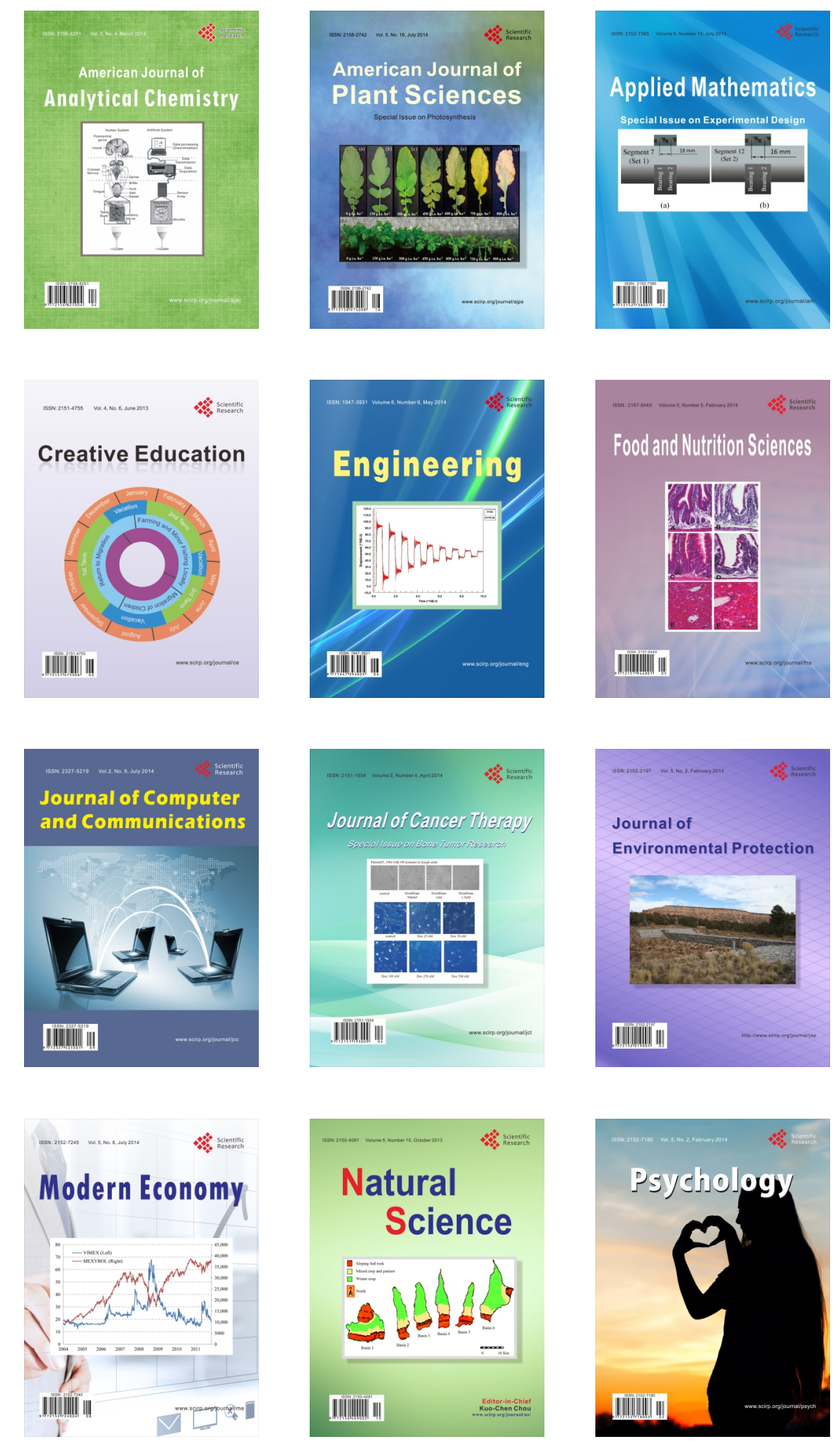